\title{
Concentration of fine needle aspirates similar to molecular method improves sensitivity of the diagnosis of tuberculous lymphadenitis in Addis Ababa, Ethiopia
}

\author{
Olifan Zewdie ${ }^{1,2^{*}}$, Tamrat Abebe ${ }^{2}$, Adane Mihret ${ }^{2}$,Eyob Hirpa ${ }^{3}$ and Gobena Ameni ${ }^{4}$
}

\begin{abstract}
Background: Tuberculous lymphadenitis (TBLN) diagnosis has been a true challenge solely by clinical evidence in developing countries, due to limited the diagnostic facility on hand. However, the availability and affordability of available diagnostic tools in resource-limited settings like Ethiopia necessitates the quest for other techniques with added value over direct Z-N microscopy. Therefore, we aimed at to assess whether the concentration of lymph node aspirate similarly improves the detection rate of tuberculous lymphadenitis or not.

Materials and methods: A cross-sectional study design was conducted on 132 individual subjects presumptive for tuberculous lymphadenitis from February to October 2013 in Addis Ababa, Ethiopia. Fine needle aspirate (FNA) samples were collected from the cases and cultured on Löwenstein-Jensen ( $L J)$ slants. Identification of species and strains of mycobacteria was made by region of difference (RD) based polymerase chain reaction (PCR). Data entry and statistical analyses were performed by SPSS version 20. The confidence level of 95\% was used for statistical significance.

Result: A total of 132 study subjects were included in our study. Of these $56.1 \%$ (74/132) were positive for M. tuberculosis on culture. The detection rate of direct smear microscopy and the concentration method were 29.5 and $65.2 \%$ respectively. The sensitivity of direct smear microscopy was $43.2 \%$, for concentrated smear microscopy 94.5\%, for PCR 93.2\% and for cytomorphology $95.4 \%$. The level of agreement of concentrated ZN smear microscopy was 0.62 which was very similar with kappa of 0.58 of molecular (PCR) technique. AFB positivity by the concentration method and molecular method was increased in caseous aspirates as compared to purulent and hemorrhagic aspirates though it was not statistically significant ( $p$-value $=0.18)$ and $(p=0.62)$ respectively.
\end{abstract}

Conclusion: The concentration of FNA (Fine Needle Aspirate) aspirates for acid-fast smear microscopy similarly improves the sensitivity of acid fast bacilli in diagnosing of TBLN.

Keywords: Smear concentration, PCR, TBLN, FNA, Addis Ababa

\footnotetext{
* Correspondence: olifangdr@gmail.com

'Department of Medical Laboratory Sciences, College of Medical and Health

Sciences, Wollega University, PO Box 395, Nekemte, Ethiopia

2Department of Microbiology, Immunology and Parasitology, School of

Medicine, Addis Ababa University, Addis Ababa, Ethiopia

Full list of author information is available at the end of the article
} 


\section{Background}

M. tuberculosis, one of the most harmful human pathogens worldwide, causes considerable morbidity and mortality [1]. Tuberculosis is also a major public health problem in Ethiopia which ranks 7th among the 22 High Burden Tuberculosis Countries (HBTCs) and ranks 2nd EPTB cases globally [2]. The prevalence of all forms of TB is estimated at 261 per 100,000 population, leading to an annual mortality rate of 64 per 100,000 [3]. In Ethiopia, extra pulmonary tuberculosis (EPTB) accounted for $34.8 \%$ of TB cases with more than $80 \%$ of cases presenting as $\mathrm{Tu}$ berculosis lymphadenitis cases [4]. Even though early case detection and treatment is one of the pillars of the TB control program, extra pulmonary tuberculosis is a significant true challenge in its different clinical presentation. The challenges are due to the significant proportion of clinical samples, a low number of bacilli in EPTB specimens and their slow growth rate which reduce the sensitivity of conventional diagnostic methods [5].

Definitive diagnosis of tuberculous lymphadenitis is often difficult as most of the available techniques are low either in sensitivity or specificity as compared to culture. Clinical features, though indicative of tuberculous etiology, are not adequate for making a definitive diagnosis [6]. Fine-needle aspiration (FNA) has become a widely used diagnostic tool and it remains one of the most rapid and cost-effective diagnostic methods of tuberculous lymphadenitis but is characterized by low specificity [7]. The primary method for the diagnosis of tuberculosis is Ziehl Neelsen staining. Although the method is specific and rapid; the technique has low sensitivity in the detection of tubercle bacilli in various clinical specimens $[8,9]$. The identification of tubercle bacilli by culture is required for the ultimate proof of mycobacterial infection. However, due to unavailability of laboratory equipment and safety procedures, the method is not practiced in resource-poor settings. [10, 11]. Nucleic acid methods in the specific target like Region of Difference are currently employed for rapid identification of mycobacteria from both cultured and uncultured clinical specimens [12].

Previous studies have shown that liquefaction of sputum and lymph node aspirate with some liquefaction chemical reagents and then concentrated by centrifugation before acid-fast staining significantly improves the sensitivity of smear microscopy [13, 14]. The aim of present study is to evaluate whether treatment with some chemical reagents followed by concentration by centrifugation of lymph node aspirate improves the sensitivity of smear microscopy similarly with compared to the molecular method.

\section{Methods}

\section{Patient recruitment and study settings}

This was a cross-sectional study conducted from February to October 2013 in Ethiopia at Tikur Anbessa Specialized Hospital (TASH) and Alem Tena Higher Clinic (ATHC). One hundred and thirty-two clinically suspected TBLN patients, who were referred to Pathology laboratory of TASH and ATHC and who gave their consent for the examination of fine needle aspirates (FNAs), were included in this study. Demographic data for all patients were collected using a pre structured questionnaire by trained clinical nurses. Patients who were below 18 years old and who were taking anti-TB treatment at the time of sample collection were excluded from the study.

\section{Sample collection and processing}

FNA samples were collected by a pathologist from the affected nodes. Briefly, the swollen area was cleaned with $70 \%$ alcohol and then 21 gauge needle was inserted into the mass. After removing the needle, drops of aspirate were placed on a clean slide for FNA cytology, thereafter, leftover FNA samples were added aseptically into sterile universal tubes in phosphate buffer saline $\mathrm{pH} 7.2$ at $4{ }^{\circ} \mathrm{C}$, and transported in cold chain to TB laboratory at Aklilu Lemma Institute of Pathobiology (ALIPB), for further microbiological and molecular analysis.

\section{FNA concentration for detection of M.tubeculosis}

FNA specimens were homogenized with $0.85 \%$ normal saline and then decontaminated and digested with $4 \%$ $\mathrm{NaOH}$ for $15 \mathrm{~min}$ at room temperature, the samples then concentrated by centrifugation at $3000 \mathrm{~g}$ for 15 min at Aklilu Lemma Institute of Pathobiology Mycobacteriology Laboratory [3]. The sediment was neutralized with $2 \mathrm{~N}$ hydrochloric acid, using phenol red as an indicator. After centrifugation, the supernatant was decanted carefully and 2-3 drops of the sediment were placed on a clean slide and stained with Ziehl-Neelsen (ZN) staining.

\section{Culture}

After homogenization the sediment of processed samples was inoculated onto the conventional LowensteinJensen (LJ) egg slant medium, containing $0.6 \%$ sodium pyruvate and glycerol and incubated at $37^{\circ} \mathrm{C}$ for at least 6 weeks, with weekly observation for the presence of mycobacterial colonies. Microscopic examination of the colonies was performed using Ziehl-Neelsen stain to select AFB-positive isolates.

\section{DNA extraction}

Mycobacterial genomic DNA was extracted as previously described by Van sooligen et al. [15] with minor 
modifications. Briefly, $0.2 \mathrm{ml}$ of the FNA material was centrifuged at $1200 \mathrm{rpm}$ for $20 \mathrm{~min}$ and the supernatant was discarded. Thereafter the pellet was re-suspended in $500 \mu \mathrm{l} \mathrm{TE} .50 \mu \mathrm{l}$ of $10 \mathrm{mg} / \mathrm{ml}$ of lysozyme (Boehringer Mannheim, Mannheim, Germany) was added and mixed well before incubation for $1 \mathrm{~h}$ at $37{ }^{\circ} \mathrm{C}$. The lysozymetreated samples were incubated at $65{ }^{\circ} \mathrm{C}$ for $10 \mathrm{~min}$ in the presence of $6 \mu \mathrm{l}$ of $10 \mathrm{mg}$ of proteinase $\mathrm{K} / \mathrm{ml}$ (Boehringer Mannheim) and $70 \mu \mathrm{l}$ of $10 \%$ sodium dodecyl sulfate (Boehringer Mannheim, Mannheim, Germany) $0.100 \mu \mathrm{L}$ of $5 \mathrm{M} \mathrm{NaCl}$ and $80 \mu \mathrm{L}$ of pre-warmed CTAB/ $\mathrm{NaCl}$ (Cetyl trimethyl ammonium bromide in sodium chloride) (Merck, p.a) solution was added, and vortexed until the liquid content become "milky" followed by incubation at $65{ }^{\circ} \mathrm{C}$ for $10 \mathrm{~min}$. Extraction was performed by adding approximately 700-800 $\mu$ l of chloroform/isoamyl alcohol (24:1) (Merck, p.a) and then centrifuged for $10 \mathrm{~min}$ at $12,000 \mathrm{rpm}$. The aqueous phase was transferred to a fresh micro centrifuge tube. The DNA precipitate was obtained by adding $450 \mu \mathrm{l}$ of isopropanol (Merck, p.a) to the aqueous phase. After storage for $1 \mathrm{~h}$ at $-20{ }^{\circ} \mathrm{C}$, the DNA was collected by centrifugation at $12,000 \mathrm{rpm}$ for $15 \mathrm{~min}$, washed with cold $70 \%$ ethanol, and centrifuged for $5 \mathrm{~min}$ at 12,000 rpm. Most of the supernatant was removed and placed at room temperature for $15 \mathrm{~min}$ to evaporate all the ethanol. Finally, the pellet was resuspended in 1xTE and stored at $-20^{\circ} \mathrm{C}$ until the PCR assay was performed.

\section{Region of difference 9-based deletion typing by PCR}

Identification of mycobacterial species causing TBLN was performed using PCR based RD9 and RD4 deletion typing based PCR were used as described earlier [16], using RD9_FlankF, IntR and FlankR and RD4 flankF, IntR and flankR primers. The primers used were RD9_FlankF, IntR and FlankR and RD4 flankF, IntR and flankR primers, and their sequences were published earlier [17]. PCR was performed using a standard thermocycler (VWR Thermo cycler, VWR International, UK). The reaction mixture RD9-based PCR consisted of $10 \mu \mathrm{l}$ Hot-StarTaqMaster Mix (Qiagen, Crawley, UK), $7.1 \mu \mathrm{l}$ Qiagen water, $0.3 \mu \mathrm{l}$ of each of the three primers $(100 \mathrm{mM})$ and $2 \mu \mathrm{l}$ DNA template giving a total volume of $20 \mu \mathrm{l}$. M. tuberculosis H37Rv and M. bovis, and Qiagen water were used as positive and negative controls, respectively. The reaction mixture was then heated using Thermal Cycler PCR machine (VWR International, UK) using the following amplification program: $95{ }^{\circ} \mathrm{C}$ for $10 \mathrm{~min}$ for enzyme activation; $95^{\circ} \mathrm{C}$ for $1 \mathrm{~min}$ for denaturation; $61{ }^{\circ} \mathrm{C}$ for $0.5 \mathrm{~min}$ for annealing; $72{ }^{\circ} \mathrm{C}$ for $2 \mathrm{~min}$ for extension, involving 35 cycles all in all; and final extension at $72{ }^{\circ} \mathrm{C}$ for $10 \mathrm{~min}$. The product was electrophoresed using the Agarose Gel Electrophoresis System (Bio-Rad, Hernel Hempstead, UK) in $1.5 \%$ agarose gel in $1 \times$ EDTA running buffer. Ethidium bromide at a ratio of 1:10, 100 base pair DNA ladder, and orange $6 \times$ loading dye were used in gel electrophoresis. The gel was visualized in a Multilmage TM light cabinet using Alpha Innotech, version 1.2.0.1 (Alpha Innotech Corporation, Cannock, UK). The results were interpreted as M. tuberculosis (RD9 present) when a band size of 396 bp was observed and as either M. bovis or $M$. africanum (absence of RD9) when the band size of 575 bp was observed. The absence of RD9 was further differentiated by RD4 typing; interpreted as M. bovis (RD4 deleted) when the band size of 446 was observed whereas presence of RD4 (M. tuberculosis or $M$. africanum) when the band size of 335 was detected.

\section{Data analysis}

All demographic and laboratory data were entered, cleared and analyzed using SPSS version 20 (SPSS Inc., Chicago, IL, USA). The Categorical data was analyzed using the Chai-Square $\left(X^{2}\right)$ or the fisher exact test that assumes when expected cell size $n>5$. A $p$-value $\leq 0.05$ was considered statistically significant. The confidence level of 95\% was used for statistical significance. The criteria for cyto- histopathological diagnosis was epithelioid cell granulomas with or without multinucleate giant cells, with or without necrosis and caseous necrosis without granuloma. Accuracy is defined as the closeness of the measured result to the true value and culture used as a gold standard in our study.

\section{Result}

Socio-demographic characteristics of the study subjects

A total of 132 clinically suspected TBLN patients who fulfilled the inclusion criteria were included in our study. The study participants' age ranged from 18 to 71 years old with the mean age of 31.4 years and +/- 12.2 standard deviation. More than half $52.2 \%(69 / 132)$ of presumptive TBLN cases were found in the age group of 18 to 27 years. Out of 132 suspected patients, 55.3\% (73/ $132)$ were females. The majority $66.6 \%(88 / 132)$ of clients were presumptive TBLN presented with cervical lymphadenopathy. Lymph node condition was reported as hard matted in $47.7 \%(63 / 132)$ of the cases.

\section{Detection rate}

Cytomorphological features of FNAC consistent with tuberculosis lymphadenitis were reported in $83.3 \%(110 /$ 132) of the examined smeared specimens. All FNA samples were processed for bacterial growth on Lowenstein-Jensen (L-J) media, of which 56.1\% (74/132) were confirmed as TBLN. According to species identification protocol, no non-tuberculous mycobacterium was reported. M.tubeculosis were detected in 39 FNA making the detection rate of direct $\mathrm{ZN}$-smear $29.5 \%$ and the 
Table 1 Efficacy and Kappa values of diagnostic yield of different diagnostic methods used in the diagnosis of TBLN

\begin{tabular}{|c|c|c|c|c|c|c|}
\hline \multirow[t]{2}{*}{ Diagnostics Methods } & \multicolumn{3}{|l|}{ Culture } & \multirow{2}{*}{$\begin{array}{l}\text { Sensitivity } \\
(\%)\end{array}$} & \multirow{2}{*}{$\begin{array}{l}\text { Specificity } \\
(\%)\end{array}$} & \multirow[t]{2}{*}{ Kappa (95\% Cl) } \\
\hline & & Positive $n(\%)$ & Negative $n(\%)$ & & & \\
\hline \multirow[t]{2}{*}{ Direct ZN staining } & Positive $(n=39)$ & $32(82.1)$ & $7(17.9)$ & \multirow[t]{2}{*}{43.2} & \multirow[t]{2}{*}{87.9} & \multirow[t]{2}{*}{$0.32(0.21-0.41)$} \\
\hline & Negative $(n=93)$ & $42(45.2)$ & $51(54.8)$ & & & \\
\hline \multirow[t]{2}{*}{ Concentrated ZN staining } & Positive $(n=86)$ & $70(81.4)$ & $16(18.6)$ & \multirow[t]{2}{*}{94.5} & \multirow[t]{2}{*}{72.4} & \multirow[t]{2}{*}{$0.62(0.57-0.81)$} \\
\hline & Negative $(n=46)$ & $4(8.7)$ & $42(89.3)$ & & & \\
\hline \multirow[t]{2}{*}{ Direct PCR } & Positive $(n=91)$ & $69(75.8)$ & $22(24.2)$ & \multirow[t]{2}{*}{93.2} & \multirow[t]{2}{*}{62.1} & \multirow[t]{2}{*}{$0.58(0.41-0.71)$} \\
\hline & Negative $(n=41)$ & $5(12.2)$ & $36(87.8)$ & & & \\
\hline \multirow[t]{2}{*}{ Cytology } & Positive $(n=110)$ & $71(64.5)$ & $39(35.5)$ & \multirow[t]{2}{*}{95.4} & \multirow[t]{2}{*}{32.8} & \multirow[t]{2}{*}{$0.26(0.22-0.046)$} \\
\hline & Negative $(n=22)$ & $3(13.6)$ & $19(86.4)$ & & & \\
\hline
\end{tabular}

detection rate of concentration method of $\mathrm{ZN}$-smear become $65.2 \%$. Thus the concentration methods detect 47 extra patients with an increased yield of 35.6\% (47/132) over direct $\mathrm{ZN}$ method. The $\mathrm{ZN}$ concentration method detection rate was very similar to a molecular method which detects $68.9 \%(91 / 132)$ of FNA samples.

\section{Accuracy and level of agreement of different diagnostic modalities}

The different diagnostic accuracy and level of agreement were compared. Of 74 culture positive FNA 32 (43.2\%) and $70(94.5 \%)$, were smear positive on direct ZN staining and on NALC-NaOH concentration methods respectively. Moreover, 69 (93.2\%) and 71 (95.4\%) showed positive signal on direct PCR and indicated cytomorphological features consistent with TB respectively. The sensitivity of $\mathrm{ZN}$ concentration method and direct PCR was ideal compared measures; however, the sensitivity of direct smear microscopy was less than those methods $43.2 \%$. The level of agreement of concentrated $\mathrm{ZN}$ smear microscopy was 0.62 which was ideal compared measures with a kappa of 0.58 of direct PCR in detection of TB against culture. The low sensitivity of direct $\mathrm{ZN}$ staining was also reflected in the low 0.32 kappa agreement measure Table 1.

\section{Cytomorphological characteristics}

A total of $91.7 \%(121 / 132)$ presumptive FNA analyzed consistent the cytological picture of tuberculous lymphadenitis based on the mentioned cytomorphological criteria. Of these, the concentration method and the direct PCR method detect AFB ideal compared measures Table 2.

Cytomorphological consistent of tuberculosis lymphadenitis for all case was clearly described. Of these, the most 42\% (56/132) showed epithelioid cell with necrosis and the least cases $11.4 \%(15 / 132)$ were reported in polymorphs with necrosis. Other cytological patterns had shown in. While overall increased AFB positivity rate was observed in necrosis aggregated epithelioid cells, decreased rate was observed in polymorphs cells with necrosis Table 3 .

We tried to associate and compared some factors in ZN-staining concentrated method and molecular method. AFB positivity by the concentration method and molecular method was increased in caseous aspirates as compared to purulent and hemorrhagic aspirates though it was not statistically significant $(p$-value $=0.18)$ and $(p=0.62)$ respectively. However, AFB positivity by the concentration method and molecular method was statistically associated with tenderness of the lymph node $(p$-value $=0.04)$ and $(p$-value $=0.02)$ respectively Table 4 .

\section{Discussion}

The diagnosis of TBLN has been a true challenge solely by clinical evidence in the developing countries due to the limited diagnostic facility on hand [18]. If the diagnosis of TBLN can be established faster, and the diagnostic process becomes less cumbersome for the

Table 2 Relation of FNAC result on Concentrated and direct ZN smear positivity compared with direct PCR positivity

\begin{tabular}{|c|c|c|c|c|c|c|c|}
\hline \multirow[t]{2}{*}{ FNAC result } & \multirow{2}{*}{$\begin{array}{l}\text { Total cases \% } \\
(N)\end{array}$} & \multicolumn{2}{|l|}{ Direct ZN staining } & \multicolumn{2}{|c|}{ Concentrated ZN staining } & \multicolumn{2}{|l|}{ Direct PCR } \\
\hline & & Positive \% (n/N) & Negative \% (n/N) & Positive \% (n/N) & Negative \% (n/N) & Positive \% (n/N) & Negative \% (n/N) \\
\hline TBLN & $91.7(121 / 132)$ & $32.2(39 / 121)$ & $67.8(82 / 121)$ & $69.0(83 / 121)$ & $31.4(38 / 121)$ & $71.9(87 / 121)$ & $28.1(34 / 121)$ \\
\hline Suppurative abscess & $3(4 / 132)$ & $0.0(0 / 4)$ & $100(4 / 4)$ & $25.0(1 / 4)$ & $75.0(3 / 4)$ & $25.0(1 / 4)$ & $75.0(3 / 4)$ \\
\hline Reactive LN & $2.3(3 / 132)$ & $0.0(0 / 3)$ & $100(3 / 3)$ & $66.7(2 / 3)$ & $33.3(1 / 3)$ & $66.7(2 / 3)$ & $33.3(1 / 3)$ \\
\hline Others diagnosis & $3(4 / 132)$ & $0.0(0 / 4)$ & $100(4 / 4)$ & (0.0) $0 / 4$ & $100(4 / 4)$ & $25.0(1 / 4)$ & $75(3 / 4)$ \\
\hline
\end{tabular}


Table 3 Cyto-morphological patterns in AFB positivity (concentrated and direct smears) compared with PCR and culture positivity

\begin{tabular}{llllll}
\hline Cytological patterns & Cases \% $(\mathrm{n} / \mathrm{N})$ & $\begin{array}{l}\text { Direct ZN } \\
\text { positive \% }(\mathrm{n} / \mathrm{N})\end{array}$ & $\begin{array}{l}\text { Concentrated ZN } \\
\text { positive \% (n/N) }\end{array}$ & $\begin{array}{l}\text { Direct PCR } \\
\text { positive \% (n/N) }\end{array}$ & $\begin{array}{l}\text { Culture } \\
\text { positive \% (n/N) }\end{array}$ \\
\hline Epithelioid cell with necrosis & $42(56 / 132)$ & $44.6(25 / 56)$ & $73.2(41 / 56)$ & $77.0(42 / 56)$ & $62.5(35 / 56)$ \\
Epithelioid cell without necrosis & $31.8(42 / 132)$ & $9.5(4 / 42)$ & $61.9(26 / 42)$ & $69.0(29 / 42)$ & $66.7(28 / 42)$ \\
Necrosis without epithelioid cell & $14.4(19 / 132)$ & $31.6(6 / 19)$ & $52.6(10 / 19)$ & $63.2(12 / 19)$ & $57.9(11 / 19)$ \\
Polymorphs with necrosis & $11.4(15 / 132)$ & $20.0(3 / 15)$ & $33.3(5 / 15)$ & $33.3(5 / 15)$ & $66.6(10 / 15)$ \\
\hline
\end{tabular}

patient, PCR may reduce a delay both in the diagnosis and in the start of treatment [19]. But in resource limiting setting like Ethiopia, the method is not feasible. Thus, the method concentrating FNA sample that has similar accuracy with the molecular method but with the low cost required.

The study has shown that the concentration of lymph node aspirate followed by centrifugation was significantly improves the sensitivity of smear microscopy compared measures to the molecular method [20]. The present finding demonstrates comparable result with the study done in Bangladesh [20]. The detection rate of the direct method was $29.5 \%$ and it increased to $65.2 \%$ in the concentration method which was ideal compared measures to $68.9 \%$ in the molecular method. The increased smear positivity by the concentration method which was ideally a comparable measure to PCR was given the evidence to the higher density of bacilli per microscopic field and increase clarity of microscopic field.

The low detection rate and the low sensitivity of direct $\mathrm{ZN}$-staining of the present study was consistent with earlier finding [21]. The low bacilli found in the lymph node aspirates and more than 10,000 bacilli/ml of sample required to be positive on smear microscopy could be the main factor for the decreased sensitivity and low detection rate of direct smear. Though the sensitivity of this method was found to be relatively low, the high

Table 4 Factors assessed for associated with positivity when considering concentration and molecular method in Addis Ababa

\begin{tabular}{|c|c|c|c|c|c|c|c|c|c|}
\hline \multirow[t]{2}{*}{ Variables } & & \multicolumn{2}{|c|}{ Concentration method \% (n/N) } & \multirow[t]{2}{*}{$\mathrm{OR}[95 \% \mathrm{Cl}]$} & \multirow[t]{2}{*}{$P$-value } & \multicolumn{2}{|c|}{ PCR method \% (n/N) } & \multirow[t]{2}{*}{ OR $[95 \% \mathrm{Cl}]$} & \multirow[t]{2}{*}{$P$-value } \\
\hline & & Positive & Negative & & & Positive & Negative & & \\
\hline \multirow[t]{2}{*}{$\overline{S e x}$} & Female & $63.0(46 / 73)$ & $37.0(27 / 73)$ & 1 & 0.6 & $67.8(40 / 59)$ & $32.2(19 / 59)$ & 1 & 0.8 \\
\hline & Male & $67.8(40 / 59)$ & $32.2(19 / 59)$ & $1.2[0.6-2.5]$ & & $69.8(51 / 73)$ & $30.1(22 / 73)$ & $0.9[0.4-1.9]$ & \\
\hline \multirow[t]{5}{*}{ Age } & $18-27$ & $65.2(45 / 69)$ & $34.8(24 / 69)$ & $0.8[0.3-2.0]$ & & $66.7(46 / 69)$ & $33.3(23 / 69)$ & $0.5[0.2-1.5]$ & \\
\hline & $28-37$ & $71.4(20 / 28)$ & $28.6(8 / 28)$ & $1.9[0.7-5.0]$ & 0.6 & $78.6(22 / 28)$ & $21.4(6 / 28)$ & $1.7[0.6-4.4]$ & 0.2 \\
\hline & $38-47$ & $50.0(11 / 22)$ & $50(11 / 22)$ & $1.9[0.214 .2]$ & & $54.5(12 / 22)$ & $45.5(10 / 22)$ & $0.7[0.1-6.7]$ & \\
\hline & $48-57$ & $50.0(2 / 4)$ & $50.0(2 / 4)$ & $0.2[0.03-2.0]$ & & $75.0(3 / 4)$ & $25.0(1 / 4)$ & $0.2[0.02-2.1]$ & \\
\hline & $\geq 58$ & $88.9(8 / 9)$ & $11.1(1 / 9)$ & 1 & & $88.9(8 / 9)$ & $11.1(1 / 9)$ & 1 & \\
\hline \multirow[t]{2}{*}{ Resident } & Rural & $66.1(39 / 59)$ & $33.9(20 / 59)$ & 1 & 0.8 & $69.8(51 / 73)$ & $30.2(22 / 73)$ & 1 & 0.8 \\
\hline & Urban & $64.4(47 / 73)$ & $35.6(26 / 73)$ & $0.9[0.5-1.9]$ & & $67.8(40 / 59)$ & $32.2(19 / 59)$ & $1.1[0.5-2.3]$ & \\
\hline \multirow[t]{2}{*}{ Tenderness of LN } & Non-tender & $73.2(48 / 65)$ & $26.8(17 / 65)$ & 1 & 0.04 & $78.5(51 / 65)$ & $21.5(14 / 65)$ & 1 & 0.02 \\
\hline & Tender & $56.7(38 / 67)$ & $43.3(29 / 67)$ & $0.5[0.2-0.9]$ & & $59.7(40 / 67)$ & $40.3(27 / 67)$ & $0.4[0.2-0.8]$ & \\
\hline \multirow[t]{3}{*}{ Increasing of LN } & Slow & $71.9(23 / 32)$ & $28.1(9 / 32)$ & 1 & 0.6 & $81.3(26 / 32)$ & $18.7(6 / 32)$ & 1 & 0.03 \\
\hline & Moderate & $62.2(46 / 74)$ & $37.8(28 / 74)$ & $0.7[0.2-2.3]$ & & $68.9(51 / 74)$ & $31.1(23 / 74)$ & $0.3[0.1-0.8]$ & \\
\hline & Fast & $65.4(17 / 26)$ & $34.6(9 / 26)$ & $1.2[0.4-2.9]$ & & $53.8(14 / 26)$ & $46.2(12 / 26)$ & $0.5[0.2-1.3]$ & \\
\hline \multirow[t]{3}{*}{ No of LN } & Single & $74.0(34 / 46)$ & $26.0(12 / 46)$ & 1 & 0.3 & $82.6(38 / 46)$ & $17.2(8 / 46)$ & 1 & 0.1 \\
\hline & Few & $60.3(41 / 68)$ & $39.7(27 / 68)$ & $0.5[0.1-1.8]$ & & $61.7(42 / 68)$ & $38.2(26 / 68)$ & $0.3[0.1-1.1]$ & \\
\hline & Many & $61.1(11 / 18)$ & $38.9(7 / 18)$ & $1.0[0.3-3.0]$ & & $61.1(11 / 18)$ & $38.9(7 / 18)$ & $0.9[0.3-2.8]$ & \\
\hline \multirow[t]{2}{*}{ Mobility LN } & Non-mobile & $61.4(59 / 96)$ & $38.6(37 / 96)$ & 1 & 0.15 & $64.6(62 / 96)$ & $35.4(34 / 96)$ & 1 & 0.1 \\
\hline & Mobile & $75.0(27 / 36)$ & $25.0(9 / 36)$ & $0.5[0.2-1.2]$ & & $80.5(29 / 36)$ & $19.5(7 / 36)$ & $0.4[0.2-1.1]$ & \\
\hline \multirow[t]{3}{*}{ Types of aspirate } & Purulent & $60.0(49 / 86)$ & $40(37 / 86)$ & 1 & & $63.9(55 / 86)$ & $36.1(31 / 86$ & 1 & \\
\hline & Caseous & $91.9(34 / 37)$ & $8.1(3 / 37)$ & 1.8 [0.6-11.3] & 0.18 & $83.8(31 / 37)$ & $16.2(6 / 37)$ & $1.4[0.4-5.6]$ & 0.62 \\
\hline & Hemorrhagic & $33.3(3 / 9)$ & $66.7(6 / 9)$ & $0.12[0.1-0.4]$ & & $55.5(5 / 9)$ & $44.5(4 / 9)$ & $03[0.1-0.9]$ & \\
\hline
\end{tabular}


specificity of this method allowed to the clinician in of initiation of anti-TB treatment which eliminated antibiotic and anti-TB trials for the patient.

Positivity rate of tuberculosis lymphadenitis was increased in direct and concentrated microscopy and molecular method like PCR when a cytomorphological characteristic of FNAC was epithelioid cell with necrosis aggregates. By the concentration and molecular methods, there is an increase in AFB positivity from 61.9 to $73.2 \%$ and 69.0 to $77.0 \%$ respectively in those with epithelioid cell aggregates without necrosis to when epithelioid cell aggregates and present with necrosis. Our finding is in line with studies done by others that showed increased positivity for different cytomorphological patterns in smears revealing necrosis aggregates with epithelioid cell $[22,23]$. This finding can be explained by the fact that a predominantly necrotic reaction of the tubercle contains more bacilli.

The current study of TBLN showed that the concentration method (91.9\%) and on molecular method (83.8\%) was high positive yield result as compared to hemorrhagic and purulent aspirates though it didn't show any significance. This might be due to the fact that the caseous aspirate perforates the deep fascia and escapes into the superficial fascia resulting in collar abscess formation and believed to contain large numbers of bacilli in an advanced stage of the disease [21].

\section{Conclusion}

In conclusion, the low sensitivity of the clinico- microbiological techniques in extra pulmonary specimens have opens the door for the increased use of molecular methods for their high sensitivity and specificity but, their evaluation in low-income countries have been limited. To overcome this problem, the method which has similar sensitivity with PCR should be used. To the effect of this, an increased sensitivity observed in smear concentration method ideally compare measures with PCR can be recommended.

\footnotetext{
Abbreviations

CAS: Central Asia; DNA: Deoxy ribonucleic acid; DR: Direct repeat; DST: Drug susceptibility test; ETH: Ethiopia; FNA: Fine needle aspirate; INH: Isoniazid; LJ: Lowenstein-Jensen; LPA: Line probe assay; MDRTB: Multi-drug resistance tuberculosis; PCR: Polymerase chain reaction; RMP: Rifampin; RNA: Ribonucleic acid; SIT: Spoligo international type; TB: Tuberculosis; TBLN: Tuberculosis Iymphadenitis; WHO: World Health Organization; WT: Wild type
}

\section{Acknowledgements}

We would like to thank Addis Ababa University and Akililu Lemma institute of Pathobiology for their cooperation and all study subjects who were volunteered to participate in this study. Moreover, we would like to thank Pathology department at Addis Ababa University and Alem Tena higher clinic staffs for their encouragement.

\section{Funding}

Addis Ababa University and Akililu Lemma institute of Pathobiology support us financially only for data collection.

\section{Availability of data and material}

The datasets generated and/or analyzed during the current study are not publicly available because we have another manuscript for analysis but, the data are available on reasonable request.

\section{Authors' contributions}

OZ was the primary researcher, conceived the study, designed, participated in sample collection, performed laboratory experiments, conducted data analysis and drafted the manuscript for publication. TA, AM, EH and GA reviewed the initial and final manuscript. All authors read and approved the final manuscript.

\section{Competing interests}

The authors declare that they have no competing interests.

\section{Consent for publication}

Not applicable in this section.

\section{Ethics approval and consent to participate}

Ethical permission was obtained from the Ethical and review committee of Department of Microbiology, Immunology and Parasitology, College of Health Science, Addis Ababa University. Written informed consent was obtained from each participant before collection of samples.

\section{Author details}

1Department of Medical Laboratory Sciences, College of Medical and Health Sciences, Wollega University, PO Box 395, Nekemte, Ethiopia. ²Department of Microbiology, Immunology and Parasitology, School of Medicine, Addis Ababa University, Addis Ababa, Ethiopia. ${ }^{3}$ School of Veterinary Medicine, Wollega University, Nekemte, Ethiopia. ${ }^{4}$ Aklilu Lemma Institute of

Pathobiology, Addis Ababa University, Addis Ababa, Ethiopia.

Received: 1 September 2016 Accepted: 6 January 2017

Published online: 14 January 2017

\section{References}

1. Mohammad A, Hossein S. Current trends in molecular epidemiology studies of Mycobacterium tuberculosis. Biotech and Molec Biology Rev. 2007:2(5):108-15.

2. World Health Organization. Global Tuberculosis Control 2014. Geneva, Switzerland:who.int/tb/publications/global report/

3. Federal Ministry of Health of Ethiopia. Health Sector Development Programme IV. Annual performance report 2010. Addis Ababa, Ethiopia

4. Biadglegne F, Tesfaye $W$, Anagaw B, Tessema B, Debebe $T$, et al. Tuberculosis lymphadenitis in Ethiopia. Jpn J Infect Dis. 2013;66:263-8.

5. TB prevalence surveys: a handbook. Geneva, World Health Organization, 2013 (WHO/HTM/TB/2013 report)

6. Derese $Y$, Hailu E, Assefa T, Bekele Y, Mihret A, et al. Comparison of PCR with standard culture of fine needle aspiration samples in the diagnosis of tuberculosis lymphadenitis. J Infect Dev Ctries. 2012;6:53-7.

7. Aljafari AS, Khalil EA, Elsiddig KE, El Hag IA, Ibrahim ME, et al. Diagnosis of tuberculous lymphadenitis by FNAC, microbiological methods and PCR: a comparative study. Cytopathology. 2004;15:44-8.

8. Bouakline SH, Vincensini JP, Giacuzzo V, Lagrange PH, Herrmann JL. Rapid diagnosis of extra pulmonary tuberculosis by PCR: impact of sample collection and DNA extraction. J Clin Microbiol. 2003;41:2323-9.

9. Ergete $W$, Bekele $A$. Acid fast bacilli in fine needle aspiration smear from tuberculous patients. Eth J Heal Devep. 2000;14:99-104.

10. Hung J, Shen M, Sun Y. Epidemiological analysis of extra pulmonary tuberculosis in Shanghai. Artic in Chinese. 2000;23:606-8.

11. American Thoracic Society (ATS). Diagnostic standards and classification of tuberculosis in adults and children. Am J Respir Crit Care Med. 2000; 161:1376-95.

12. Eisenach KD, Cave MD, Bates JH, Crawford JT. Polymerase Chain Reaction amplification of a repetitive DNA sequence specific for M. tuberculosis. J Infect Dis. 1990:161:977-81.

13. Wilkinson D, Sturm AW. Diagnosing tuberculosis in a resource poor setting: the value of sputum concentration. Trans R Soc Trop Med Hyg. 1997:91:420-1. 
14. Annam V, Karigoudar MH, Yelikar BR. Improved microscopical detection of acid-fast bacilli by the modified bleach method in lymphnode aspirates. Indian J Pathol Microbiol. 2009;52:349-52.

15. Van Soolingen D, Hoogenboezem T, de Haas PE, Hermans PW, Koedam MA, Teppema KS, Brennan PJ, Besra GS, Portaels F, Top J, Schouls LM, van Embden JD. A novel pathogenic taxon of the M. tuberculosis complex, Canetti: characterization of an exceptional isolate from Africa. Int J Syst Bacteriol. 1997:47:1236-45.

16. Brosch R, Gordon SV, Marmiesse M, et al. A new evolutionary scenario for the M. tuberculosis complex. Proc Natl Acad Sci U S A. 2002;99:3684-9.

17. Berg S, Firdessa R, Habtamu M, et al. The burden of mycobacterial disease in Ethiopian cattle: implications for public health. PLoS One. 2009;4:e5068.

18. Kurabachew M, Enger O, Sandaa RA, Skuce R, Bjorvatn BA. Multiplex PCR assay for genus, group and species specific detection of Mycobacteria. Diagnostic Microbiol Infec Dis. 2004:49:99-104

19. Kivihya-Ndugga $L$, van Cleeff $M$, Juma $E$, et al. Comparison of $P C R$ with the routine procedure for diagnosis of tuberculosis in a population with high prevalences of tuberculosis and human immunodeficiency virus. I Clin Microbiol. 2004;42:1012-5.

20. Runa F, Yasmin M, Hoq MM, Begum J, Rahman ASMM, Ahsan CR. Molecular versus conventional methods: clinical evaluation of different methods for the diagnosis of tuberculosis in Bangladesh. J Microbiol Immunol Infect. 2011;44:101-5

21. Iwnetu $R$, van den Hombergh J, Woldeamanuel Y, Asfaw M, Gebrekirstos $C$, Nugusie Y, Bekele T, Ashenafi S, Seyoum B, Melaku K, Asefa A. Is tuberculous lymphadenitis over-diagnosed in Ethiopia? Comparative performance of diagnostic tests for mycobacterial lymphadenitis in a high burden country. Scand J Infect Dis. 2009;41:462-8.

22. Bezabih M, Mariam DW, Selassie SG. Fine Needle aspiration cytology of suspected tuberculous lymphadenitis. Cytolpathology. 2002;13:284-90.

23. Paliwal N, Thakur S, Mullick S, Gupta K. Fine needle aspirate cytology in tuberculous lymphadenitis: experience from tertiary referral centre. Indian J Tuberc. 2011:58:102-7.

\section{Submit your next manuscript to BioMed Central and we will help you at every step:}

- We accept pre-submission inquiries

- Our selector tool helps you to find the most relevant journal

- We provide round the clock customer support

- Convenient online submission

- Thorough peer review

- Inclusion in PubMed and all major indexing services

- Maximum visibility for your research

Submit your manuscript at www.biomedcentral.com/submit 